\title{
Cluster of HIV Infections Attributed to Unsafe Injection Practices - Cambodia, December 1, 2014-February 28, 2015
}

\author{
Mean Chhi Vun ${ }^{1}$; Romeo R. Galang²; Masami Fujita 3 ; William Killam4; Runa Gokhale²; John Pitman ${ }^{5}$; Dejana Selenic ${ }^{5}$; Sovatha Mam¹; \\ Chandara Mom ${ }^{1}$; Didier Fontenille ${ }^{6}$; Francois Rouet ${ }^{6}$; Saphonn Vonthanak ${ }^{7}$; Roka Cluster Investigation Team
}

In December 2014, local health authorities in Battambang province in northwest Cambodia reported 30 cases of human immunodeficiency virus (HIV) infection in a rural commune (district subdivision) where only four cases had been reported during the preceding year. The majority of cases occurred in residents of Roka commune. The Cambodian National Center for HIV/AIDS (acquired immunodeficiency syndrome), Dermatology and Sexually Transmitted Diseases (NCHADS) investigated the outbreak in collaboration with the University of Health Sciences in Phnom Penh and members of the Roka Cluster Investigation Team. By February 28, 2015, NCHADS had confirmed 242 cases of HIV infection among the 8,893 commune residents, an infection rate of $2.7 \%$. Molecular investigation of the HIV strains present in this outbreak indicated that the majority of cases were linked to a single HIV strain that spread quickly within this community. An NCHADS case-control study identified medical injections and infusions as the most likely modes of transmission. In response to this outbreak, the Government of Cambodia has taken measures to encourage safe injection practices by licensed medical professionals, ban unlicensed medical practitioners, increase local capacity for HIV testing and counseling, and expand access to HIV treatment in Battambang province. Measures to reduce the demand for unnecessary medical injections and the provision of unsafe injections are needed. Estimates of national HIV incidence and prevalence might need to be adjusted to account for unsafe injection as a risk exposure.

The Roka Cluster Investigation Team initiated an investigation to confirm cases, identify risk factors, and recommend control strategies. Data from antiretroviral therapy (ART) sites and registers of community-based HIV/AIDS care programs were reviewed to exclude persons with existing HIV diagnoses. Specimens that had tested HIV-positive by HIV rapid test kit were laboratory confirmed using an enzyme immunoassay (Serodia, Fujirebio Diagnostics, Japan). Specimens were also tested for antibody to hepatitis C (anti-HCV) and hepatitis B surface antigen (HBsAg). A case-control study was undertaken to identify risk factors associated with HIV infection. Controls were selected from commune residents who tested HIV-negative at the time of the study and were matched by age, sex, and place of residence. To describe the number and size of HIV infection clusters among the outbreak cases, phylogenetic analysis was performed on blood specimens from case patients by the Institut Pasteur du Cambodge. Limiting-antigen (LAg) Avidity assay testing was performed to identify recent infection.

The index patient was a resident of Roka commune with tuberculosis, aged 74 years, who received a diagnosis of HIV infection on November 12, 2014. (Figure) Two of the index patient's family members also tested positive for HIV during the same period. The family alleged that the infections were linked to medical injections received from an unlicensed health practitioner. These allegations triggered a surge in demand for HIV testing by other commune residents. During November 2014-February 2015, a total of 2,045 commune residents underwent HIV testing. Overall, 242 confirmed HIV cases were identified, including $52(22 \%)$ in children aged $<14$ years, and $51(21 \%)$ in adults aged $>60$ years. One hundred fifty cases $(62 \%)$ were in females. Four women aged $>60$ years and one girl aged 7 months died after their HIV diagnoses; the causes of death are unknown. As of January 19, among 102 patient specimens tested, 72 (70.6\%) were positive for anti-HCV, and eight (7.8\%) were positive for HBsAg. Current national data are not available for comparison; however, population prevalence of anti-HCV and HBsAg in the neighboring province of Siem Riep are estimated at $5.8 \%$ and $4.6 \%$, respectively (1). An investigation of the provincial blood transfusion center ruled out blood transfusion as a source of infection in this cluster. Preliminary results from the casecontrol study indicated that cases were nearly five times as likely as controls to have received an intravenous or intramuscular injection, and four times as likely as controls to have received an intravenous infusion during the preceding 6 months.

Phylogenetic analyses of the $\mathrm{C} 2-\mathrm{V} 3$ region of the HIV-1 gp120 gene, and related protease and reverse transcriptase genes demonstrated clustering of HIV viral strains among the outbreak cases and similarity between strains identified in the outbreak and other strains in Southeast Asia. Preliminary incidence assay results (Sedia LAg Avidity enzyme immunoassay, Sedia Biosciences Corporation, Portland, Oregon) suggested that $30 \%$ of infections in this outbreak could be classified as having occurred within the 130 days preceding specimen collection.

Concurrent to the case-control study, NCHADS implemented confirmatory HIV testing, conducted community outreach, and supported the scale-up of voluntary HIV testing and counseling in the commune and in the provincial capital (Battambang City). ART services were established at the Roka 
FIGURE. Number of persons ( $\mathrm{N}=242)$ infected with human immunodeficiency virus, by date of diagnosis - Roka Commune, Cambodia, November 9, 2014-February 28, 2015

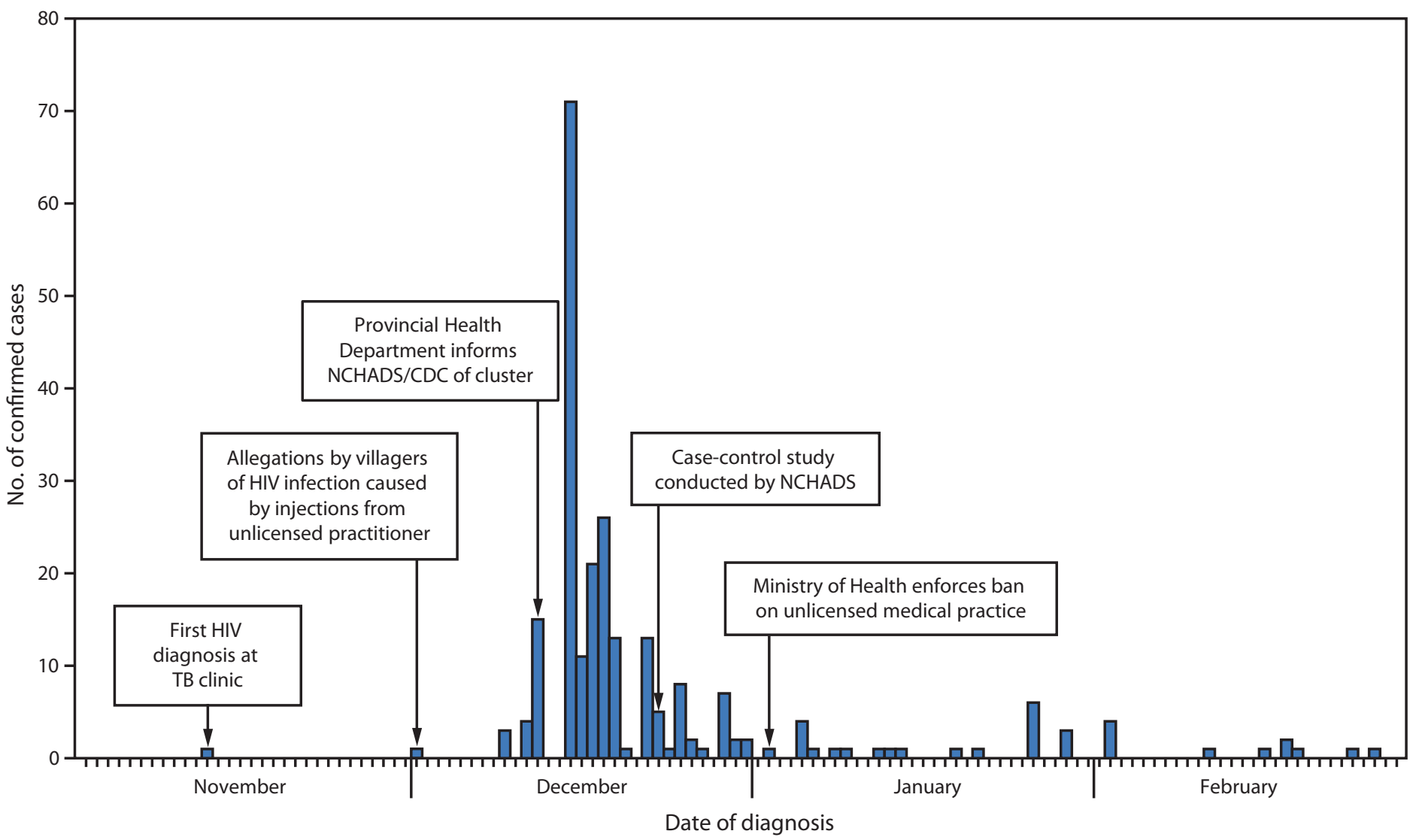

Abbreviations: $\mathrm{HIV}=$ human immunodeficiency virus; NCHADS = National Center for HIV/AIDS, Dermatology and Sexually Transmitted Diseases; TB = tuberculosis.

village health center, complementing existing ART services at the Battambang regional hospital. By January 16, 2015, a total of 207 patients, including 179 adults and 28 children (86\% of the 242 identified patients with HIV) had initiated ART; the remaining patients were registered in pre-ART care.

A majority of the confirmed cases in this outbreak were from a population not associated with commercial sex work, men who have sex with men (MSM), or injection drug use, the primary risk factors driving Cambodia's HIV epidemic (2). The clustering of HIV cases across age groups and other evidence indicating high demand for medical injections in Cambodia further support the likelihood of transmission via injection, intravenous infusion, or other invasive medical procedures (3).

\section{Discussion}

Cambodia has successfully reduced national HIV incidence and contained HIV prevalence among commercial sex workers, MSM, and persons who inject drugs. However, this outbreak highlights the risk for HIV transmission in the general population through unsafe medical injections (2). HIV transmission by unsafe medical injections has not historically been prioritized in Cambodia's national HIV prevention strategy, which has focused on transmission associated with sex and injection drug use, and, to a lesser extent, blood safety.

Demand for medical injections among Cambodian adults is high, averaging 2.6 injections per person per year, compared with countries such as Vietnam (1.5 injections per person per year), India (2.0), and Nepal (1.2) (4). On average, women in Cambodia receive more injections per year (3.3 per person per year, weighted $95 \%$ confidence intervals $[\mathrm{CI}]=3.1-3.6)$ than men $(1.9,95 \% \mathrm{CI}=1.7-2.2)$, but in some provinces, women receive as many as 5.9 injections per year on average (5). The proportion of injections administered with reused equipment in this cluster is unknown; however, a 2013 study estimated $5.5 \%$ reuse in the Western Pacific region (4). Analyses of Cambodia's 2005 Demographic Health Survey data indicate that 14,618 HIV-negative persons received an average of 2.0 $(95 \% \mathrm{CI}=1.8-2.1)$ medical injections per person per year, whereas $84 \mathrm{HIV}$-positive persons received an average of 7.2 $(95 \% \mathrm{CI}=2.6-11.8)$ medical injections per person per year. Despite this substantial difference, it is not known whether HIV infection resulted from medical injections, or whether 
persons living with HIV receive more medical injections because they are sicker. Furthermore, a portion of the association among HIV-infected persons might be confounded by injections received for other sexually transmitted infections ( $G$ ).

The per-act risk for HIV transmission from unsafe medical injections has been estimated among select populations and within nosocomial outbreak settings globally. The risks for transmission among persons who inject drugs and share needles and among health care workers with occupational exposure through percutaneous needle-stick injuries were estimated at 63 and 23 per 10,000 acts, respectively (7); however the authors reported wide confidence intervals because of a lack of uniformity in these exposures. A recent outbreak of HIV infections among persons who inject drugs in a rural community in the United States also illustrated the explosive outbreak potential when HIV is introduced into settings where contaminated needles are shared (8).

Nosocomial HIV outbreaks, as recently demonstrated in Kyrgyzstan, have demonstrated the potential for overuse of medical injections to cause outbreaks in low-risk populations in countries with HIV epidemics that are concentrated in certain high-risk groups (9). In these nosocomial outbreaks, HIV transmission risk per injection with HIV-contaminated equipment has been estimated to be as high as $2 \%-7 \%(7,10)$. In 2004 , it was estimated that $1 \%-5 \%$ of new HIV infections in sub-Saharan Africa might be associated with unsafe medical injections ( $($ ).

The findings in this report are subject to at least three limitations. First, case identification might be limited to persons who sought HIV testing because of perceived risk of infection related to an unlicensed practitioner rather than with an unsafe injection, leading to a possible underestimation of the total number of cases. Second, findings from the case-control study support an association between medical injection and HIV infection; however, a causal relationship could not be established. Finally, the type and frequency of procedures and the type of equipment used are unknown, limiting ability to identify specific practices (e.g., contamination of multidose medication vials, and sharing of needle or infusion equipment) associated with HIV infection.

The Cambodian government has issued guidance to local health departments to increase enforcement of medical licensing regulations and holds monthly meetings to monitor progress toward this goal. Planning is underway to expand HIV surveillance and evaluate medical injection risk factors elsewhere in Cambodia. Future interventions will seek to reduce public demand for medical injections nationally, and raise health care worker awareness about infection control as well as noninjectable alternatives.

Cambodia's current national HIV prevalence and incidence estimates are based on models that do not include risk factors associated with unsafe injections or blood transfusion. Given

\begin{abstract}
Summary
What is already known about this topic?

Unsafe medical injection practices have been reported in Cambodia during the last decade. Current national human immunodeficiency virus (HIV) prevalence estimates do not include HIV transmission risk associated with unsafe injection or blood transfusion. HIV testing and surveillance in Cambodia are focused on high risk groups, including men who have sex with men, persons who inject drugs, and commercial sex workers.

What is added by this report?

The largest cluster of new HIV infections ever attributed to unsafe injections among a general population was reported in a rural area of Cambodia; $2.7 \%$ of residents were infected. The outbreak was detected after increased demand for HIV testing by residents who perceived themselves to be at risk after exposure to an unlicensed provider of injections and intravenous infusions.

What are the implications for public health practice?

HIV prevention strategies that target specific populations often do not consider the risk for HIV transmission via unsafe injections in the general population. Further studies are needed to clarify HIV prevalence in general populations where HIV risk perception is low; quantify the risk for other bloodborne infections (e.g., hepatitis C) via unsafe injections; understand public demand for medical injections; and improve health care workers' injection practices in the public and private sectors. Measures to reduce both the demand for unnecessary medical injections and the provision of unsafe injections are needed.
\end{abstract}

the high prevalence of medical injection use in Cambodia, the contribution of medical injection overuse to Cambodia's national HIV burden might be higher than estimated. Efforts should be made to educate health care workers and communities at large on safe injection practices to reduce the demand for unnecessary medical injections and increase injection safety. National HIV prevention strategies should be expanded to monitor unsafe injections as a mode of transmission. Globally, a need exists for tools to estimate HIV risk in low-prevalence countries where substantial proportions of the population are regularly exposed to unnecessary and potentially unsafe injections.

\section{Acknowledgments}

Students, faculty, staff members, Cambodia University of Health Sciences; FHI 360, North Carolina; ARV Users Association, Cambodia; AIDS Healthcare Foundation, the Netherlands; Khmer Soviet Friendship Hospital, Cambodia; Dr. Dora Warren, CDC Cambodia Country Director (2009-2015).

\footnotetext{
${ }^{1}$ Cambodia National Center for HIV/AIDS, Dermatology and STD; ${ }^{2}$ Epidemic Intelligence Service, CDC; ${ }^{3}$ World Health Organization, Cambodia; ${ }^{4}$ Division of Global HIV/AIDS, CDC, Phnom Penh, Cambodia; ${ }^{5}$ Division of Global HIV/AIDS, CDC; ${ }^{6}$ Institut Pasteur du Cambodge, Phnom Penh, Cambodia; ${ }^{7}$ Cambodia University of Health Sciences.
}

Corresponding author: Romeo R. Galang, RGalang@cdc.gov, 404-639-6387. 


\section{References}

1. Yamada H, Fujimoto M, Svay S, et al. Seroprevalence, genotypic distribution and potential risk factors of hepatitis $\mathrm{B}$ and $\mathrm{C}$ virus infections among adults in Siem Reap, Cambodia. Hepatol Res 2015;45:480-7. http://dx.doi.org/10.1111/hepr.12367.

2. National AIDS Authority. Cambodia country progress report: monitoring progress towards the targets of the 2011 UN Political Declaration on HIV and AIDS. Phnom Penh, Cambodia: National AIDS Authority; 2014. http://www.unaids.org/sites/default/files/country/documents//KHM_ narrative_report_2014.pdf.

3. Vong S, Perz JF, Sok S, et al. Rapid assessment of injection practices in Cambodia, 2002. BMC Public Health 2005;5:56-62. http://dx.doi. org/10.1186/1471-2458-5-56.

4. Pépin J, Abou Chakra CN, Pépin E, Nault V. Evolution of the global use of unsafe medical injections, 2000-2010. PLoS One 2013;8:e80948. http://dx.doi.org/10.1371/journal.pone.0080948.

5. National Institute of Statistics; Directorate General for Health; ICF Macro. Cambodia demographic and health survey 2010. Phnom Penh, Cambodia: National Institute of Statistics, Directorate for General Health; Calverton, Maryland: ICF Macro; 2011. http://dhsprogram.com/pubs/pdf/FR249/ FR249.pdf.
6. Schmid GP, Buvé A, Mugyenyi P, et al. Transmission of HIV-1 infection in sub-Saharan Africa and effect of elimination of unsafe injections. Lancet 2004;363:482-8. http://dx.doi.org/10.1016/S0140-6736(04)15497-4.

7. Patel P, Borkowf CB, Brooks JT, Lasry A, Lansky A, Mermin J. Estimating per-act HIV transmission risk: a systematic review. AIDS 2014;28:1509-19. http://dx.doi.org/10.1097/QAD.0000000000000298.

8. Conrad C, Bradley HM, Broz D, et al. Community outbreak of HIV infection linked to injection drug use of oxymorphone-Indiana, 2015. MMWR Morb Mortal Wkly Rep 2015;64:443-4.

9. Utyasheva L. Kyrgyzstan: nine health care workers guilty of negligence causing HIV transmission among children. HIV AIDS Policy Law Rev 2008;13:48-9.

10. Gisselquist D, Upham G, Potterat JJ. Efficiency of human immunodeficiency virus transmission through injections and other medical procedures: evidence, estimates, and unfinished business. Infect Control Hosp Epidemiol 2006;27:944-52. http://dx.doi.org/10.1086/506408. 